\title{
ON CANONICAL CONSTRUCTIONS. III
}

\author{
MURRAY GERSTENHABER
}

In the first paper in this series a structure $S$ was called canonically reconstructible from a structure $T$ provided $S$ was canonically isomorphic to a structure $T^{\prime}$ derived from $T$. The principle proposition of the paper was the scholium which asserted: If a structure $S$ contains a substructure $T$ and if $S$ is canonically reconstructible from $T$, then every automorphism of $T$ may be extended uniquely to an inner automorphism of $S$ (relative to the derivation of $S$ from $T$ ). To illustrate the concepts it was proved in that paper that every automorphism of the group of permutations $\Sigma(N)$ of a set $N$ (finite or infinite) was inner in the group theoretic sense except when $N$ has 6 elements. Let $A(N)$ now denote the subgroup of $\Sigma(N)$ generated by all 3-cycles, i.e., all permutations moving exactly 3 elements of $N$. This will be called the alternating group on $N$. It will here be shown that the set $N$ can be canonically reconstructed from any subgroup $G$ of $\Sigma(N)$ which contains $A(N)$ when $N$ has 4,5 , or more than 6 elements. It follows by the principles of On canonical constructions, I, that every automorphism of $G$ can then be extended uniquely to an automorphism, i.e., permutation, of $N$, which in turn induces an inner automorphism of $\Sigma(N)$. This is trivially true when $N$ has 1 or 2 elements. Therefore, it is the case, except when $N$ has cardinality 3 or 6, that every automorphism of $G$ can be extended uniquely to an inner automorphism of $\Sigma(N)$, which is the principle theorem of this paper. ${ }^{1}$

Substructures as derived structures. $A$ substructure $R$ of a structure $S$ is construable as a structure derived from $S$ if it is canonically characterisable within $S$; such a substructure will be called characteristic. The characterization may be given by a statement of the form, " $R$ is the unique substructure of $S$ with the property. ..." For example, when $N$ is a finite set $A(N)$ may be considered as a structure derived from $\Sigma(N)$, being the unique subgroup of index two. (This gives an example of a derived structure which is a group but in which an inner automorphism as a derived structure is not necessarily inner in the group theoretic sense.) The object of this sec-

Received by the editors March 29, 1955.

1 When $N$ has 3 elements the extension is possible but not unique. For finite $N$ the theorem is due to $\mathrm{O}$. Hölder [3]. 
tion is to demonstrate that for any finite or infinite $N$, if $G$ is a subgroup of $\Sigma(N)$ which contains $A(N)$ then $A(N)$ may be considered as a structure derived from $G$. Let $n$ henceforth denote the cardinality of $N$. The matter is already settled for finite $n$. For $n \geqq 5$ (and therefore in particular for all infinite $n$ ) it will be shown that $A(N)$ is the only normal simple subgroup of $G$ not reduced to the identity, and the matter will then be settled for all $n$. For the remainder of this section it is presumed that $n \geqq 5$.

It is evident that $A(N)$ is normal, and it remains to prove that it is simple and is the only normal simple subgroup of $G$ not reduced to the identity. (That $A(N)$ is simple is known [1] but for completeness we reproduce a proof here.) If $X$ is any subset of the set $N$ on which $\Sigma(N)$ acts, then the group of all permutations leaving fixed all elements outside $X$ will be denoted by $\Sigma(X)$, and its alternating subgroup by $A(X)$. We shall consider only finite $X$ with at least 5 elements; then $A(X)$ is simple. The union of $A\left(X_{1}\right)$ and $A\left(X_{2}\right)$ is contained in $A\left(X_{1} \cup X_{2}\right)$, and $A(N)$ is the union of all the $A(X)$. If $A^{\prime}$ is a normal subgroup of $A(N)$ not reduced to the identity then it has a nontrivial intersection with some $A(X)$. This intersection, being a nontrivial normal subgroup of $A(X)$, must be all of $A(X)$, so $A^{\prime} \supseteq A(X)$. Similarly $A^{\prime}$ contains $A\left(X^{\prime}\right)$ for all $X^{\prime} \supseteq X$, so $A^{\prime} \supseteq A(N)$ and $A(N)$ is therefore simple.

To show that $A(N)$ is the only normal simple subgroup of $G$ not reduced to the identity it is sufficient now to show that if $B$ is any other such subgroup then $B$ has a nontrivial intersection with $A(N)$. Let $b$ be any element of $B$ other than the identity. It can not commute with every element of $A(N)$, whence there exists an $a$ in $A(N)$ such that $c=a b a^{-1} b^{-1} \neq 1$. Writing $c$ as $\left(a b a^{-1}\right) b^{-1}$ it follows that $c$ is in $B$ since $B$ was assumed normal. Similarly $c$ is in $A(N)$. Therefore, $B \cap A(N)$ is not reduced to the identity, whence it follows that $B \supseteq A(N)$ and the proposition is proved: $A(N)$ is the only nontrivial normal simple subgroup of any subgroup $G$ of $\Sigma(N)$ which contains $A(N)$. This in turn proves the proposition that $A(N)$ is construable as a structure derived from $G$. (It of course demonstrates that $A(N)$ is a characteristic subgroup of $G$.)

Characterization of a 3-cycle within $A(N)$. If $N$ has fewer than 6 elements, then inside $A(N)$ a 3-cycle is completely characterized by the fact that it is an element of order 3. When $N$ has 6 elements the characterization is known to be impossible. For the remainder of this section it will therefore be assumed that $N$ has at least 7 elements, and for such an $N$ a set of properties will be given which completely characterize a three-cycle within $A(N)$. 
If $G$ is a group and $H$ a subgroup, then $H$ will be said to be submaximal if there exists a proper subgroup of $G$ properly containing $H$ and if every such subgroup is maximal in $G$. For any element $a$ in $A(N)$, let $C(a)$ denote the group of elements of $A(N)$ commuting with it. It will be shown that if $a$ is a 3-cycle then $C(a)$ is a submaximal subgroup of $A(N)$, and is of order $(n-3) ! 3$ when $n$ is finite $(n=$ cardinality of $N)$. Further, it will be shown that if $a^{\prime}$ is an element of $A(N)$ of order 3 , if $C\left(a^{\prime}\right)$ is submaximal, and if for finite $n$, $C\left(a^{\prime}\right)$ is of order $(n-3) ! 3$, then $a^{\prime}$ is a 3 -cycle.

By the carrier of a permutation in $\Sigma(N)$ will be meant the set of elements actually moved by the permutation. The elements of $N$ will henceforth be called points. $A$ permutation will be called finite or infinite according as the number of points in its carrier is finite or infinite. If $X$ is any subset of $N$, the set of all permutations whose carriers are contained in $X$ has been denoted by $\Sigma(X)$. The finite permutations in $\Sigma(X)$ form a smaller subgroup $\Sigma^{\prime}(X)$ which in turn contains the alternating group $A(X)$ on $X$. The carrier of a subgroup $G$ of $\Sigma(N)$ will be the union of the carriers of its elements. If $G$ and $H$ are subgroups of $\Sigma(N)$ whose carriers are disjoint, then we may form their internal direct product $G H$ in $\Sigma(N)$. If $X$ and $Y$ are any two disjoint subsets of $N$ then we may form in particular $\Sigma^{\prime}(X) \Sigma^{\prime}(Y)$, $\left[\Sigma^{\prime}(X) \Sigma^{\prime}(Y)\right] \cap A(N)$ (which will be denoted by $A(X, Y)$ ), and $A(X) A(Y)$. Each of these groups contains the next one mentioned, but the inclusions are not necessarily strict, since if $Y$ contains but one point then $A(X, Y)$ and $A(X) A(Y)$ coincide, both being just $A(X)$. However, if $X$ and $Y$ each contain at least two points, then $A(X, Y)$ does strictly contain $A(X) A(Y)$, and the index of the latter group in the former is two. For an element $a=a_{x} a_{y}$ of $\Sigma^{\prime}(X) \Sigma^{\prime}(Y)$ can be in $A(N)$ if and only if either $a_{x}$ and $a_{y}$ are both even, in which case $a$ is in $A(X) A(Y)$, or if $a_{x}$ and $a_{y}$ are both odd, and all elements of the second sort clearly lie in the same coset of $A(X) A(Y)$.

Under certain conditions a subgroup of $A(N)$ of the form $A(X) A(Y)$ is submaximal, a result for which the following lemma is needed. (It will be understood that a product $a b$ is the effect of performing first $b$, then $a$.)

Lemma. Let $X$ and $Y$ be disjoint and $X \cup Y=N$, and suppose $X$ and $Y$ each have at least three elements. Let a be any element of $A(N)$ not in $A(X, Y)$ and suppose $a(X) \neq Y$. Then given any other element $b$ in $A(N)$, there is an element $c$ in the group $G$ generated by $a$ and $A(X) A(Y)$ such that bc is in $A(X, Y)$.

Proof. If $b$ maps no element of $X$ into $Y$, then $b$ is already in 
$A(X, Y)$ and $c$ may be taken to be the identity. It is therefore sufficient to show that if $b$ maps $r$ elements of $X$ into $Y$ and $r$ is positive, then an element $c^{\prime}$ can be found in $G$ such that $b c^{\prime}$ maps at most $r-1$ elements of $X$ into $Y$. To this end observe first that there can be found an element $a^{\prime}$ in $G$ mapping exactly one element of $X$ into $Y$ (and hence also exactly one element of $Y$ into $X$ ). For by the assumption on $a$, neither $Y \cap a^{-1}(X)$ nor $Y \cap a^{-1}(Y)$ is empty, and there is therefore an element $d$ of $A(Y)$ carrying exactly one element of $a^{-1}(X)$ onto $a^{-1}(Y)$. One may then take $a^{\prime}=a d a^{-1}$. Suppose now that $x_{1}$ is a point of $X$ carried into $Y$ by $b ; b\left(x_{1}\right)=y_{1}$. There must also be a point of $Y$ carried into $X$ by $b, b\left(y_{1}^{\prime}\right)=x_{1}^{\prime}$. (It is immaterial that $x_{1}^{\prime}$ may coincide with $x_{1}$, or $y_{1}^{\prime}$ with $y_{1}$.) Let $x_{2}^{\prime}$ be that point of $X$ carried into $Y$ by $a^{\prime}$ and $y_{2}$ that point of $Y$ carried by it into $X$. If $a^{\prime}\left(x_{2}{ }^{\prime}\right)$ were $y_{1}^{\prime}$ and $a^{\prime}\left(y_{2}\right)$ were $x_{1}$, then $a^{\prime}$ would serve as the desired $c^{\prime}$. Since the sets $X$ and $Y$ each have, by assumption, at least three elements, $A(X)$ and $A(Y)$ act transitively on them. There exists, therefore, an element $a_{x}$ of $A(X)$ carrying $a^{\prime}\left(y_{2}\right)$ into $x_{1}$ and an element $a_{y}$ of $A(Y)$ carrying $a^{\prime}\left(x_{2}^{\prime}\right)$ into $y_{1}^{\prime}$. Choose then $c^{\prime}=a_{x} a_{y} a^{\prime}$, and the lemma is proved.

The foregoing lemma demonstrates in particular that $A(X, Y)$ is a maximal subgroup of $A(N)$. One may now derive the following:

LEMma. Let $X$ and $Y$ be disjoint, each contain at least three elements, and let $X \cup Y=N$. If either the cardinality of $N$ is not finite, or if it is finite and the cardinality of $X$ is different from that of $Y$, then $A(X) A(Y)$ is a submaximal subgroup of $A(N)$.

Proof. If $N$ is infinite or if the cardinality of $X$ is different from that of $Y$, then $a(X) \neq Y$ for any element $a$ in $N$, so the hypothesis of the preceding lemma is satisfied. Suppose now that $H, K$ are subgroups of $N, H \supset K \supset A(X) A(Y)$, and the inclusions are proper. Then it must be proved that $H=A(N)$. If $K=A(X, Y)$, then the matter is at an end since $A(X, Y)$ is maximal. Otherwise there exists an element $a$ of $K$ not in $A(X, Y)$. Let $G$ denote the group generated by $A(X) A(Y)$ and $a$ and let $h$ be an element of $H$ not in $K$. By the preceding lemma there exists an element $c$ of $G$ such that $h c$ is an element $d$ of $A(X, Y)$. The element $d$ so obtained can not be in $A(X) A(Y)$ for $h$ would then be $c^{-1} d$, an element of $G$ and hence in $K$, against assumption. Since $c$ is in $H$, it follows that $H$ contains an element $d$ of $A(X, Y)$ not in $A(X) A(Y)$, but since the index of $A(X) A(Y)$ in $A(X, Y)$ is two, it follows that $H$ contains $A(X, Y)$. It does so properly, and since $A(X, Y)$ is maximal, $H=N$, which proves the lemma. 
Suppose now that $a$ is any 3-cycle in $A(N)$. Let $X$ be the carrier of $a$, set $Y=N-X$, and let $C(a)$ be the group of all elements of $A(N)$ which commute with $a$. The set $X$ has 3 elements, and since we are assuming throughout that there are at least seven points in $N$ it follows that the cardinality of $Y$ is at least 4 and hence in particular is different from that of $X$. Since $C(a)=A(X) A(Y)$, it follows from the preceding lemma that $C(a)$ is a submaximal subgroup of $A(N)$. The number of elements in $A(X)$ is 3 and that in $A(Y)$, if $N$ is finite and has $n$ elements, is $n-3$. There are then $(n-3) ! 3$ elements in $C(a)$.

It has been asserted that when $n \geqq 7$, a 3-cycle $a$ in $A(N)$ is completely characterized by the properties $1 . a$ has order $3,2 . C(a)$ is a submaximal subgroup of $A(N)$, and 3 . if $n$ is finite then $C(a)$ has order $(n-3) ! 3$. It having been demonstrated that a 3 -cycle does in fact have these properties, it remains to show that if $b$ is any element of order 3 in $A(N)$ such that properties 2 . and 3 . hold for $b$, then $b$ is a 3-cycle. To this end, assume that $b$ is an element of order 3 in $A(N)$ but not a 3 -cycle. It will be shown that either property 2 . or property 3. then fails to hold.

Being a finite permutation of order $3, b$ can be written as a product of a finite number of 3 -cycles $b_{1} \cdots b_{r}(r>1)$, whose carriers $X_{1}, \cdots$, $X_{r}$ are disjoint. It will be shown first that if $X_{1} \cup \ldots \cup X_{r}$ omits more than one point of $N$ then $C(b)$ can not be submaximal in $A(N)$. Let the complement of $X_{1} \cup \cdots \cup X_{r}$ be $Y$. If $Y$ contains at least 2 points it is sufficient to show that $C(b)$ is not all of $A\left(X_{1} \cup \ldots \cup X_{r}\right) A(Y)$, since it is certainly contained in that group which is itself not maximal. But no permutation in $C(b)$ can map any element of $X_{1}$ into $X_{2}$ unless it maps all of $X_{1}$ into $X_{2}$, and there are certainly elements in $A\left(X_{1} \cdots X_{r}\right)$ which do not have this property. This proves that if $b$ is an element of $A(N)$ of order 3 but not a 3-cycle, then either the carrier of $b$ is all of $N$, omits one point, or $C(b)$ is not submaximal. This settles the case when $N$ is infinite. (In the case when $N$ is infinite therefore, an element $b$ of $A(N)$ of order three with the property that $C(b)$ is submaximal is indeed a 3-cycle.) When $N$ is finite and $C(b)$ submaximal then $X_{1} \cup \cdots \cup X_{r}$ must be all of $N$ or omit exactly one point. The number of elements in $C(b)$ is then $r ! 3^{r}$, but if the number of elements $n$ in $N$ is greater than 7 this is smaller than $(n-3) ! 3$. Of the properties $1 ., 2$., and 3., one has thus been shown to fail for a permutation $b$ which is not a 3-cycle, and the characterization of a 3-cycle is therefore complete even in the finite case.

Reconstruction of $N$. In the first two sections of this paper it has 
so far been demonstrated that if $G$ is any subgroup of $\Sigma(N)$ which contains $A(N)$ then $A(N)$ may be considered as a structure derived from $G$, and the 3-cycles have furthermore been characterized within the structure of $A(N)$ except when the number $n$ of points in $N$ is 6 . To any point $p$ in $N$ may be assigned the set $T(p)$ of all 3-cycles whose carriers contain $p$, and for $n>3$ there is a canonical one-one correspondence between the points $p$ of $N$ and the sets $T(p)$. The reconstruction of $N$, and hence the proof of the main theorem, will be at an end when the subsets of the form $T(p)$ have been characterized within $A(N)$.

When $n=4$ the complement of a set of the form $T(p)$ within the set of 3-cycles of $A(N)$ consists of a single 3-cycle and its inverse, and conversely, any 3 -cycle and its inverse constitute the complement of a set of the form $T(p)$. The sets $T(p)$ are therefore completely characterized in the case when $n=4$ and the reconstruction of $N$ is finished for that case. We may therefore assume henceforth that $n>4$.

For $n>4$, in any set $T(p)$ there exist two 3-cycles whose carriers have exactly one point of $N$ in common. Any other 3-cycle is in $T(p)$ if and only if it contains this point. To characterize $T(p)$ within $A(N)$ it is sufficient to express these assertions algebraically. The number of points which the carriers of two 3-cycles $a$ and $b$ have in common can be determined so: There are three points in common if and only if $a=b$ or $a=b^{-1}$, two points in common if and only if $a b$ does not have order 5 and $a$ and $b$ do not commute, one point in common if and only if $a b$ does have order 5 , and no points in common if and only if $a$ and $b$ commute and are not identical or inverse to each other. Therefore, a statement about the number of points which the carriers of two 3-cycles have in common may henceforth be considered an algebraic statement.

Let $p$ be any point of $N$ and $a, b$ be two 3-cycles in $T(p)$ whose carriers have exactly one point in common (namely $p$ ). The carrier of any other 3-cycle $c$ in $T(p)$ must have at least one point in common with that of $a$ and one point in common with that of $b$, but this is not sufficient for the carrier of $c$ to contain $p$ and therefore for $c$ to be in $T(p)$. Let $a$ be the permutation $(p, q, r)$ and $b$ be $(p, s, t)$. There are essentially two cases to consider. First, suppose that the carrier of $c$ has exactly one point in common with that of $a$ and exactly one point in common with that of $b$. If $c$ were in $T(p)$ then it would be of the form $(p, u, v)$ and if not in $T(p)$ it would be of the form, say, $(q, s, w)$ (here $w$ might be identical with $u$ or $v$ ). One may distinguish algebraically between the cases by observing that in 
the former the carrier of $a c a^{-1}$ is disjoint from that of $b$, but in the latter this does not hold. Second, suppose the carrier of $c$ has more than one point in common with that of $a$. If the carrier of $c$ has three points in common with that of $a$, or if it has two points in common with that of $a$ and two points in common with that of $b$, then the carrier of $c$ must contain $p$. We need then only investigate the case when the carrier of $c$ has exactly two points in common with that of $a$ and one in common with that of $b$. If $c$ is in $T(p)$ then it must be of the form $(p, q, s)$, and if not, of the form, say, $(q, r, s)$. One may distinguish algebraically between the cases by observing that in the latter $a$ is the conjugate of some power of $c$ by some power of $b$, but in the former this is not the case.

When $N$ has more than 4 elements, a set of the form $T(p)$ may therefore be fully characterized in the following fashion: 1 . The set contains two 3 -cycles $a$ and $b$ whose carriers have exactly one point in common. 2. If $c$ is any 3-cycle in $A(N)$ then $c$ is in the set if either $a$. Its carrier has exactly one point in common with that of $a$ and one in common with that of $b$, and the carrier of $a c a^{-1}$ is disjoint from that of $b$, or $b$. Its carrier has more than one point in common with, say, that of $a$ and at least one point in common with that of $b$, and $a$ is not a conjugate of some power of $c$ by some power of $b$. The characterization having also been given for $n=4$, the reconstruction of $N$ from the 3-cycles of $A(N)$ is therefore accomplished for all $N$ having 4 or more elements. Combining this with the fact that the 3 -cycles have been characterized within $A(N)$ except when $N$ has 6 elements, we have come to the end of the proof of the theorem asserted at the beginning of the paper:

TheOREM. If $G$ is any subgroup of $\Sigma(N)$ which contains $A(N)$ then every automorphism of $G$ may be extended uniquely to an inner automorphism of $\Sigma(N)$ except when $N$ has 3 or 6 elements.

(For one may observe that the only cases not discussed, namely when $n=1$ or 2 , are trivial.)

Addenda to "Canonical constructions. I" George Whaples" has pointed out that when $S$ has four elements the commutator of $(1,2)$ is contained in the group of order 8 generated by $(1,2),(3,4)$, and $(1,2)(3,4)$, hence is not maximal. In this case, therefore, one must characterize the transpositions differently in order that the proof of the theorem be complete. (The characterization given is valid for $n \geqq 5, n \neq 6$ ). An elegant characterization of transpositions involving no

\footnotetext{
${ }^{2}$ The author wishes to thank both him and W. R. Scott for their comments.
} 
counting arguments has been communicated to the author by W. R. Scott. A transposition $x$ is an element of order two with the following properties: 1 . There exists an element $y$ of order 2 such that the order of $x y^{-1} x y$ is three. 2. For no element $y$ of order two does the order of $x y^{-1} x y$ exceed three. This may be verified by direct computation (and settles the difficulty when $n=4$ ). Scott has also called the author's attention to the fact that the theorem that every automorphism of the finite symmetric group is inner $(n \neq 6)$ was proved by J. König [4], and should properly be attributed to him.

\section{REFERENCES}

1. R. Baer, Die Kompositionsreihe der Gruppe aller eineindeutigen Abbildungen einer unendlichen Menge auf sich, Studia Mathematica vol. 5 (1934) pp. 15-17.

2. M. Gerstenhaber, On canonical constructions I, Proc. Nat. Acad. Sci. U.S.A. vol. 41 (1955) pp. 233-236.

3. O. Hölder, Bildung zusammengesetzer Gruppen, Math. Ann. vol. 46 (1895) esp. pp. $340-345$.

4. J. König, Ueber rationale Funktionen von $n$ Elementen und die allgemeine Theorie der algebraischen Gleichungen, Math. Ann. vol. 14 (1879) esp. pp. 217-220.

5. J. Schreier and S. Ulam, Über die Automorphismen der Permutationsgruppe der natïrlichen Zahlenfolge, Fund. Math. vol. 28 (1937) pp. 258-260. (The theorem that every automorphism of an infinite symmetric group is inner is announced here but proved only for the countable case.)

University of Pennsylvania 